\section{A NEW SILVER CATHETER.}

HAVING frequently met with difficulty in withdrawing urine from the bladder of patients suffering from retention due to stricture, enlarged prostate, \&c., owing to the eyes of the ordinary silver catheters becoming blocked with bloodclot during their passage through the urethra, I have devised a new instrument to cope with this difficulty, whilst at the same time it possesses a great advantage over the ordinary catheter in that the inside of the instrument can be properly cleaned and rendered absolutely aseptic.

The principle of the instrument can be at once seen from the accompanying illustration and, briefly, its construction differs from the ordinary catheter in that the last half-inch of the instrument is continuous with the stilette and not with the catheter itself. By this means it can be moved in or out of position by pulling or pushing the end of the stilette and thus there is no necessity for any opening in the side of the instrument.

To use the catheter the stilette is pulled upon until the distal end is in position and the instrument is then passed into the bladder. By pushing on the end of the stilette the end of the catheter is now displaced and the urine is drawn off; the end of the instrument is then replaced and the catheter is withdrawn.

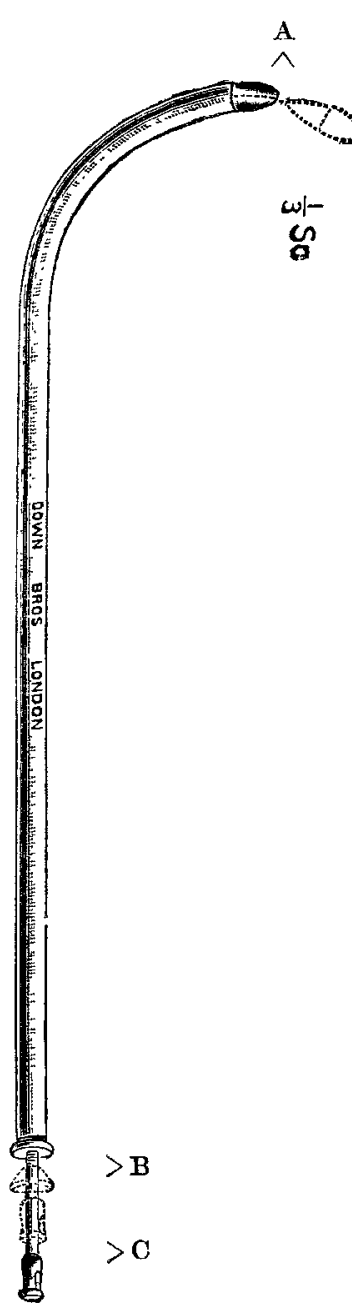

The advantages claimed for this new instrument over the present type are:-1. The absolute impossibility of the catheter becoming obstructed with bloud-clot or other foreign material during its passage into the bladder, for during this manipulation there is no opening whatever in the instrument. 2. The ease with which the flow can be regulated according to the distance the stilette is moved. 3. The rapidity with which the bladder can be emptied owing to the larger opening when the end is thoroughly dis. placed. (N.B.-In testing this instrument I found that a No. 12 ordinary silver catheter occupied 41 seconds in emptying one pint of water, whilst with this instrument (also a No. 12) it was done in 30 seconds.) 4. Supposing the eyes of the ordinary instrument to become obstructed with blood-clot in the bladder there is no way of removing it excepting by taking out the instrument altogether, whilst if the open end of this instrument becomes so blocked the clot can be broken up by alternately shutting and opening the end by means of the stilette and without taking the catheter out. 5 Its greater adaptability to asepsis, for when the instrument has been used the end is unscrewed and the small button (B) is taken off the stilette. The stilette is then withdrawn by means of the end $A$ and the inside of the catheter is thoroughly washed by a stream of water. The stilette is then replaced and the end $C$ is screwed on. A piece of gauze is afterwards passed through $\mathrm{C}$ and the stilette is again withdrawn, carrying the gauze with it which thoroughly dries the inside.

As yet I have only had the opportunity of using the instrument in a small number of cases, but in all of these it has answered admirably and has quite fulfilled all my expectations.

In conclusion I have to say that the drawing has been prepared and the instrument has been made by Messrs. Down Bros., Limited, of St. 'Thomas's-street, Borough, London, S.E., from whom it can be obtained.

STANLEY RAW,

Senior Resident Medical Officer, Sunderland Infirmary.
A SEPTOTOME FOR USE IN MOURE'S AND OTHER OPERATIONS FOR DEFLECTION OF THE TRIANGULAR CARTILAGE.

THIS instrument is a pair of detachable cutting forceps adapted from existing patterns, but specially devised to amplify the applicability of Moure's valuable operation for the rectification of the various forms of deflection or deviation of the nasal septum. The blades are modified from those of Moure's scissors from which they differ in being narrower and symmetrical; their cutting edges are but slightly divergent above and they are obtuse-somewhat probe-pointed-at the extremity. The consequence is that these blades can enter contracted fossæ and be insinuated to the required depth without endangering the folds of the limen vestibuli or the inferior turbinals, whilst the heels are sufficiently apart to clear the septum mobile. The shanks are much less curved or bowed, and at their junction with the blades there is no bending on the flat; in fact the remainder of the instrument closely follows the straight cutting forceps of Asch, but there is an addition of two powerful springs, and the result

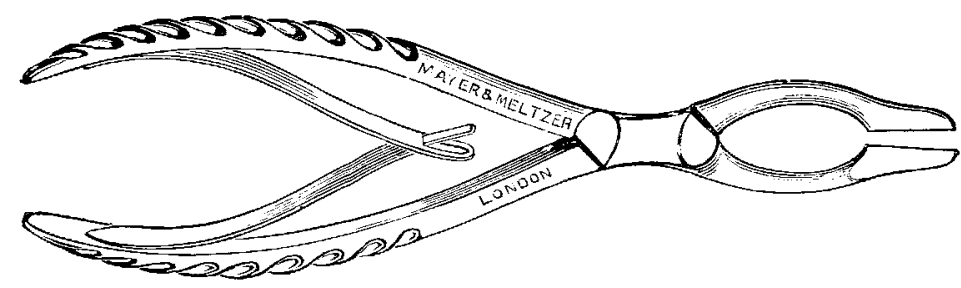

of the combination is a simple and handy septotome which works well in practice. The springs insure the disengagement of the blades after closure upon the septum, an action which greatly facilitates an extension of the primary incision, especially of the maxillary one towards the naso-pharynx, upon which, I think, so much of the success of the operation often depends. Strength is secured by the Asch form of handles, whereas the absence of an angle in the shanks, combined with symmetry in the blades, helps to maintain precision in keeping the line. For better adaptation to different requirements--such as the age of the patient, the fancy of the operator, and other conditions-the septotome can be had in three sizes, varying only in the length of the blades, No. 1 measuring half an inch, No. 2 three-quarters of an inch, and No. 3 seven-eighths of an inch $(1 \cdot 3,1 \cdot 8$, and 2.3 centimetres respectively). Of these No. 1 has, perhaps, the widest application, but the medium size answers in nearly every case, and the length from pivot to point of blade is the same in all. The septotome was made for me by Messrs. Mayer and Meltzer.

Henrietta-street, W.

L. Hemington Pegler.

\section{Looking JBack.}

FROM

THE LANCET, SATURDAY, MARCH 5, 1825.

\section{REVIEW.}

\section{A Treatise on Moxa, as applicable more particularly to Stiff} Joints. By JAMES BoyLe, Esq., Surgeon of the Middlesex Infirmary, \&c. 8vo. pp. 168. London, 1825. Callow and Wilson.

THE use of this species of cautery appears to have originated with the Japanese, by whom, and other Oriental nations, it has been much employed. The origin of the term Moxa has been a frequent subject of dispute among etymologists; but if it be not immediately derived from a word in the Japanese, nothing more than ingenious conjecture can establish its origin from other tongues. In France, the publication of Baron Larry's work on the subject procured it the sanction of many members of the profession; but, in England, little 